\title{
Weighted-Permutation Entropy Analysis of Resting State EEG from Diabetics with Amnestic Mild Cognitive Impairment
}

\author{
Zhijie Bian ${ }^{1,+}$, Gaoxiang Ouyang ${ }^{2,3,+}$, Zheng $\mathrm{Li}^{2,3}$, Qiuli Li ${ }^{4}$, Lei Wang ${ }^{4, *}$ and Xiaoli $\mathrm{Li}^{2,3, *}$ \\ 1 Department of Vascular Neurosurgery, The General Hospital of the PLA Rocket Force, Beijing 100088, China; \\ bzj2014win@163.com \\ 2 State Key Laboratory of Cognitive Neuroscience and Learning and IDG/McGovern Institute for \\ Brain Research, Beijing Normal University, Beijing 100875, China; aqqwbj@gmail.com (G.O.); \\ lz@bnu.edu.cn (Z.L.) \\ 3 Center for Collaboration and Innovation in Brain and Learning Sciences, Beijing Normal University, \\ Beijing 100875, China \\ 4 Department of Neurology, The General Hospital of the PLA Rocket Force, Beijing 100088, China; \\ liqiuli0929@163.com \\ * Correspondence: hellowanglei068@163.com (L.W.); xiaoli@bnu.edu.cn (X.L.); \\ Tel.: +86-10-6634-3392 (L.W.); +86-10-5880-2032 (X.L.) \\ + These authors contributed equally to this work.
}

Academic Editors: Osvaldo Anibal Rosso and Kevin H. Knuth

Received: 24 May 2016; Accepted: 8 August 2016; Published: 22 August 2016

\begin{abstract}
Diabetes is a significant public health issue as it increases the risk for dementia and Alzheimer's disease (AD). In this study, we aim to investigate whether weighted-permutation entropy (WPE) and permutation entropy (PE) of resting-state EEG (rsEEG) could be applied as potential objective biomarkers to distinguish type 2 diabetes patients with amnestic mild cognitive impairment (aMCI) from those with normal cognitive function. rsEEG series were acquired from 28 patients with type 2 diabetes (16 aMCI patients and 12 controls), and neuropsychological assessments were performed. The rsEEG signals were analysed using WPE and PE methods. The correlations between the PE or WPE of the rsEEG and the neuropsychological assessments were analysed as well. The WPE in the right temporal (RT) region of the aMCI diabetics was lower than the controls, and the WPE was significantly positively correlated to the scores of the Auditory Verbal Learning Test (AVLT) (AVLT-Immediate recall, AVLT-Delayed recall, AVLT-Delayed recognition) and the Wechsler Adult Intelligence Scale Digit Span Test (WAIS-DST). These findings were not obtained with PE. We concluded that the WPE of rsEEG recordings could distinguish aMCI diabetics from normal cognitive function diabetic controls among the current sample of diabetic patients. Thus, the WPE could be a potential index for assisting diagnosis of aMCI in type 2 diabetes.
\end{abstract}

Keywords: weighted-permutation entropy; resting-state EEG; neuropsychological tests; amnestic mild cognitive impairment; diabetes

\section{Introduction}

The incidence of diabetes is increasing worldwide, especially type 2 diabetes, which has been reported to result in impairment of cognitive function [1,2]. The impairment of brain function in diabetes patients has the same pathogenesis as Alzheimer's disease (AD) [3-5]. Mild cognitive impairment (MCI) lies in between or overlaps with normal health and $A D$, with cognitive function (such as learning and memory [6]) impairment but normal performance of the activities of daily living. $\mathrm{MCI}$ was reported to be a risk factor for $\mathrm{AD}$ [7], particularly amnestic MCI (aMCI). The conversion 
rate from aMCI to AD is $54 \%$ and the duration from initial diagnosis of aMCI to dementia is $28 \pm 12$ months [8]. Moreover, epidemiological data showed that the diabetic patients had 1.5 to 2.5 fold increased risk of dementia [9]. Therefore, diagnosis of aMCI among diabetic patients is very important for performing intervention to slow down the pace of aMCI conversion to AD or dementia.

Previously, the criteria of diagnosis for MCI was based on clinical analysis [10]. Neuropsychological tests and patients' histories were commonly used for diagnosing the earliest stage of MCI and AD [11]; biomarkers obtained from cerebrospinal fluid (CSF), magnetic resonance imaging (MRI), fluorodeoxyglucose positron emission tomography (FDG-PET), and ligand-based PET were all reported to be useful for the diagnosis of prodromal stages of AD [12]. However, the sensitivity and specificity of these biomarkers were different for different international databases $[13,14]$. Moreover, their clinical application is limited by the invasiveness, the high cost or the need to expose patients to radiation. Therefore, a non-invasive, easily repeatable, and cost-effective tool is needed. In comparison, EEG can play a role. It was reported that analysis of rsEEG rhythms of $\mathrm{MCI}$ and $\mathrm{AD}$ patients may be a promising approach to assess MCI [15], and scalp EEG has the potential to play a significant role as one of the earliest biomarkers for MCI and early stage AD, before clinical diagnosis [16].

Entropy as a kind of method described the probability distributions of possible states of a system [17] and revealed the complexity of the system. Different kinds of entropic algorithms have been applied to the analysis of EEG signals [18], and showed that the complexity of EEG was an important feature for distinguishing MCI or mild AD from age-matched controls [18]. PE is one method based on permutation patterns calculated entropy, and has been suggested as a complexity measure [19]. It can be used to analyse arbitrary real-world data, and suits to capture complex dynamics and abundant temporal structure embedded in biological systems [20]. For its model-free and robust to noise and artifacts, it can be applied to analyse biological data. And it has been successfully applied to EEG analysis [17], and reported to be a good biomarker for discriminating among normal elderly, $\mathrm{MCI}$ and AD [21].

However, a large amount of the amplitude information is included in EEG signals, and can be contaminated by various noises. For this reason, PE may has its limitation. An extension of PE, WPE assigns different weights to adjacent vectors that have the same permutation pattern but different amplitude variations. It retains most of the properties of PE and overcomes its shortcomings. Moreover, WPE not only captures amplitude information, but is also more robust to noise [22]. Therefore, it may be useful for detecting multi-component signals with noise or mutation.

In the previous study [23], the role of relative power and coherence was investigated in distinguishing aMCI and controls in diabetes, and the methods were linear. In this study, we try to investigate whether PE or WPE can be a potential objective complexity index of EEG to distinguish diabetics with aMCI from non-aMCI diabetic controls. There is no previous literature investigating the usefulness of WPE in general MCI. We chose to study aMCI in diabetes because it has been reported that type 2 diabetes may increase the risk of aMCI through AD related mechanisms and vascular pathology [24] and because diabetes is associated with an increased risk of MCI in elderly persons. Discovering and preventing aMCI in diabetics may reduce the risk of later $\mathrm{AD}$ onset. The effectiveness of WPE in differentiating MCI will be investigated in future work.

\section{Materials and Methods}

\subsection{Participants and Diagnostic Criteria}

The study was performed with 28 right-handed type 2 diabetes patients. The control ( 6 males and 6 females) and aMCI ( 5 males and 11 females) groups consisted of 12 patients and 16 patients, respectively. Their ages (aMCI: $69.69 \pm 2.11$, control: $73.33 \pm 1.32, p=0.16$ ), diabetes duration (aMCI: $9.25 \pm 1.47$, control: $14.00 \pm 2.79, p=0.23$ ), and educational levels (aMCI: $12.50 \pm 0.90$, control: $13.00 \pm 0.66, p=0.96$ ) were matched, but gender was not. They all satisfied the diagnosis criteria for diabetes [25]. The patients participated voluntarily and provided written informed consent 
for participation in this study. The experiment was approved by the Beijing Normal University ethics committee and conducted in accordance with the Declaration of Helsinki (1964) amended by the 64th World Medical Association General Assembly [26].

The diabetic patients all underwent neuropsychological tests, including minimum mental state examination (MMSE) [27], montreal cognitive assessment (MoCA), AVLT (AVLT-Immediate recall, AVLT-Delayed recall, AVLT-Delayed recognition) [28], WAIS-DST [29], Boston Naming Test (BNT), Trail Making Test [30], Verbal Fluency Test [31], and Daily Living Test [32]. Note that the MMSE was only used to preliminarily rule out $\mathrm{AD}$. According to Chinese government criteria, the cut-off score for absence of dementia was 24 points for people with high-school education and above, 20 points for people with primary school education, and 17 for the illiterate. The MoCA was a better screening tool for MCI in the diabetic population, because it possesses higher sensitivity (67\%) [33]. For MoCA, the cut-off score for MCI was 26 points [34].

The participants had no histories of mental illness, systemic disease, and nervous system disease which may result in cognitive impairment [23], and organic brain disease and depression were all ruled out by using MRI and the DSM IV criteria for depression [35], as reported in [23].

The inclusion criteria of diabetic aMCI patients was the diagnosis of aMCI [10] by the following symptoms: (1) subjective memory complaints reported by the patients or their family; (2) objective memory impairment evidenced by the Auditory Verbal Learning Test [28], defined by performance scores $\geq 1.5$ standard deviations below the mean value of age- and education-matched controls; (3) essentially preserved general cognitive function (assessed by MMSE and MoCA); (4) normal activities of daily living evidenced by the Activities of Daily Living Scale [32]; (5) no dementia (dementia was ruled out by DSM IV criteria for dementia [35]).

\subsection{EEG Recording and Preprocessing}

The participants were asked to close their eyes and sit relaxed in a comfortable armchair, keeping awake for $5 \mathrm{~min}$ in the Department of Neurology, The General Hospital of the PLA Rocket Force, Beijing, China. The room temperature was kept at $23 \pm 2{ }^{\circ} \mathrm{C}$ and the environment was quiet and dim. The EEG signals were recorded with a high-density 128-channel EGI system equipped with Net Amps 300 amplifiers (Electrical Geodesics Inc., Eugene, OR, USA). The recording was performed with 128-channel Geodesic Sensor Net (GSN) (Electrical Geodesics Inc., Eugene, OR, USA) using the vertex sensor $(\mathrm{Cz})$ as the reference electrode. The rsEEG signals were acquired with direct current and sampled at $1000 \mathrm{~Hz}$. All electrode impedances were kept below $50 \mathrm{k} \Omega$ according to the recommendation of EGI guidelines.

The pre-processing of the rsEEG data was performed using NetStation 4.5 software (Electrical Geodesics Inc., Eugene, OR, USA) off-line. The raw data were filtered by a $1-45 \mathrm{~Hz}$ band-pass filter, re-referenced to the average of sensor channel 57 (left mastoid process) and 100 (right mastoid process), and re-sampled to $500 \mathrm{~Hz}$ (srate $=500$ ). Artifacts (such as ocular and muscular noise) in all channels were eliminated by visual inspection of the raw EEG data, but there almost no for the patients were closed eyes and relaxed, so the segment length of data was at least $N=4500$ $(500 \mathrm{~Hz} \times 60 \mathrm{~s} \times 1.5 \mathrm{~min})$. In this study, 3-min of data from each channel were selected for further analysis, and the electrodes and areas of interest (five regions: frontal (F), left temporal (LT), central (C), right temporal (RT) and posterior (P)) were the same as described in [23].

\subsection{Entropy Analysis of EEG Data}

\subsubsection{PE}

The concept of PE was proposed by Bandt and Pompe in 2002 [19] to map a continuous time series onto a symbolic sequence. PE used existing ideas based on information theory (Shannon entropy) and could explore the local order structure of a dynamical time series as a quantitative complexity measure $[19,36]$. It transforms a given time series into a series of ordinal patterns which describe the 
order relations between the present and a fixed number of equidistant past values at a given time [37]. PE has been successfully applied to estimating the complexity of time series [37,38], e.g., EEG signals.

We consider a scalar time series $\left\{x_{1}, x_{2}, \ldots, x_{M}\right\}$, where $M$ is the length of the data. First, the series is embedded into $m$ dimensional space: $X_{j}=\left\{x_{j}, x_{j+\tau}, \ldots, x_{j+(m-1) \tau}\right\}$ for $j=1,2, \ldots, M-(m-1) \tau$, where $m$ and $\tau$ denote the embedding dimension and time delay, respectively. Then the elements of $X_{j}$ are arranged in ascending order $\left\{x_{j+\left(i_{1}-1\right) \tau} \leq x_{j+\left(i_{2}-1\right) \tau} \leq \cdots \leq x_{j+\left(i_{m}-1\right) \tau}\right\}$. In the $m$ dimensional space, each vector $X_{j}$ is mapped to a single motif out of $m$ ! possible order patterns $\pi_{i}$. Take $m=3$ for example: 6 motifs are obtained (as seen in Figure 1a). For a permutation with number $\pi_{i}$, let $f\left(\pi_{i}\right)$ denote the frequency of the $i$-th permutation in the time series. Then the probability of each order pattern can be defined as:

$$
p\left(\pi_{i}\right)=\frac{f\left(\pi_{i}\right)}{\sum_{i=1}^{m !} f\left(\pi_{i}\right)}
$$

Based on the probabilities of all permutations, the PE is defined as follows:

$$
\mathrm{H}=-\sum_{i=1}^{m !} p\left(\pi_{i}\right) \ln p\left(\pi_{i}\right)
$$

In order to make the PE value fall between 0 and 1 , we normalize $\mathrm{H}$ :

$$
0 \leq \mathrm{PE}=\mathrm{H} / \ln (m !) \leq 1
$$

The maximum value of the PE is 1 , which means that each ordinal pattern has the same probability; the minimum value is 0 , indicating that the time series is quite regular. In other words, the smaller the value of $\mathrm{PE}$ is, the more regular the time series is.

(a)

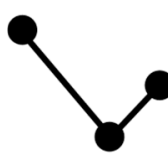

Motif \#1

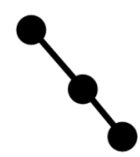

Motif \#2

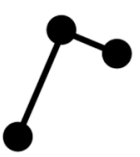

Motif \#3

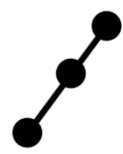

Motif \#4

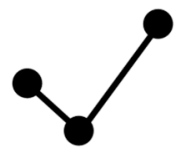

Motif \#5

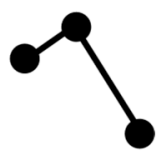

Motif \#6

(b)

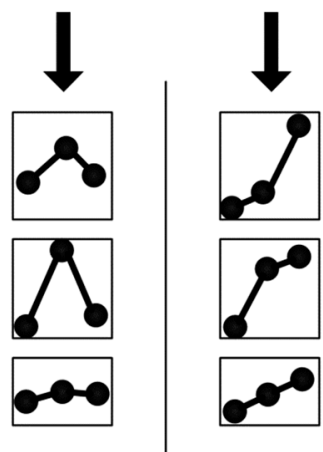

Possible Patterns

Figure 1. (a) Six permutations when $m=3$ and (b) two examples of possible $m$-dimensional vectors corresponding to the same motif (Motif \#3 and Motif \#4).

\subsubsection{WPE}

Although PE is considered a useful complexity measure of nonlinear time series, it cannot distinguish the different modes of one symbol, and the sensitivity of distinguishing background noise modes is poor. 
This is because PE only retains the order structure when extracting the ordinal patterns. This may be inconvenient for the following reasons: (1) the information in the amplitude may be lost if only the ordinal structure is extracted; (2) ordinal patterns cannot result in similar PE values when the amplitude of the time series is greatly different; and (3) noise can contribute to fluctuations of ordinal patterns in time series and the final PE value may not be weighted uniformly [39]. Figure $1 \mathrm{~b}$ shows how ordinal patterns can originate from different $m$-dimensional vectors.

To include important information when retrieving ordinal patterns of a time series, Fadlallah [22] modified the acquisition process and put forward the WPE. The main motivation was to save useful amplitude information carried by the signal. When calculating the relative frequencies for the $i$-th motif, WPE weights differently neighbouring vectors with the same ordinal pattern but different amplitude variations. Therefore, the frequency of the $i$-th permutation in the time series can be described as $f_{\omega}\left(\pi_{i}\right)=\sum_{s=1}^{S} f\left(\pi_{i}(s)\right) \cdot \omega_{i}(s), s=1,2, \ldots, S, S$ is the number of the possible patterns in the same motif, and then the weighted probability for each motif is calculated as follows:

$$
p_{\omega}\left(\pi_{i}\right)=\frac{f_{\omega}\left(\pi_{i}\right)}{\sum_{i=1}^{m !} f_{\omega}\left(\pi_{i}\right)}
$$

WPE is computed as:

$$
\mathrm{H}_{\omega}=-\sum_{i=1}^{m !} p_{\omega}\left(\pi_{i}\right) \ln p_{\omega}\left(\pi_{i}\right)
$$

When $\omega_{i}(s)=\beta \forall i \leq M-(m-1) \tau$ and $\beta>0$, WPE is equal to PE. The WPE extends the concept of PE while keeping the same expression as Shannon's entropy, hence weights are added prior to calculating the $p\left(\pi_{i}\right)$. The choice of weight values $\omega_{i}(j)$ is equivalent to selecting a specific (or combination of) feature(s) from each vector $X_{j}$. Such a feature might be different according to the context used, noting that the relation $\sum_{i} p_{\omega}\left(\pi_{i}\right)=1$ still holds. In this study, the weights were computed using variance or the energy of each neighbour's vector $X_{j} . \bar{X}_{j}$ denotes the arithmetic mean of $X_{j}$, i.e.,

$$
\bar{X}_{j}=\frac{1}{m} \sum_{k=1}^{m} x_{j+(k+1) \tau}
$$

The weight values are obtained by:

$$
\omega_{i}(s)=\frac{1}{m} \sum_{k=1}^{m}\left(x_{(j+(k-1) \tau)}-\bar{X}_{j}\right)^{2}
$$

To make the WPE value fall between 0 and 1 , we also normalize $H_{\omega}$ :

$$
0 \leq \mathrm{WPE}=\mathrm{H}_{\omega} / \ln (m !) \leq 1
$$

According to the information theory [19], when the weighted probability for each motif of random time series tends the same, the value of $\mathrm{H}_{\omega}$ was maximum tending to the logarithm of the weighted motif number $(\ln (m !))$; however, when the weighted probability for some motif is 0 in quite regular time series, the value of $\mathrm{H}_{\omega}$ is taken to be 0 . Therefore, the $\ln (m !)$ can be used to normalize the WPE, which is between 0 and 1 .

\subsubsection{Parameters for PE and WPE}

PE analyzes the local order structure of time series [19] and the WPE preserves useful amplitude information carried by the signal as well as retrieving the ordinal patterns. The calculation of PE and WPE depends on the selection of $m$. When $m$ is too small (less than 3), the scheme will work for EEG series with only very few distinct states; on the other hand, for large EEG series, a large $m$ is 
better. However, very large $m$ can result in a large number of patterns ( $m !)$, which is memory intensive. Bandt and Pompe [19] recommended $m=3,4, \ldots, 7$.

The rsEEG data was recorded in the form of a multivariate time series (number of electrodes $\times$ time), and the total length of the selected data was 3-min (srate $=500$ ), including 2 segments at most (the segments length were $N=4500$ at least). In order to track the dynamic changes of EEG smoothly, each time series was divided into overlapping segments using 10 s windows (the length of the window was $\left.N_{w}=500 \mathrm{~Hz} \times 10 \mathrm{~s}\right)$ with $90 \%$ overlap $\left(N_{\text {overlap }}=500 \mathrm{~Hz} \times 10 \mathrm{~s} \times 90 \%\right)$. Here we chose $m=4$ for the calculation of the PE and WPE. This also satisfied the condition $m !<N_{w}$, where $N_{w}$ is the window length of data. We set the time delay $\tau=1$ empirically [40]. Outliers were rejected by means of a generalized extreme studentized deviate (GESD) test [41] for all epochs in each channel. After outlier rejection, the remaining epochs in each channel were averaged and normalized. For analysis on regions of interest, the PE and WPE were obtained by averaging among channels in each region.

\subsubsection{Statistical Analysis}

In this study, the statistical analysis was conducted in GraphPad Prism5.01. The Wilcoxon rank sum test was conducted at the 5\% significance level for the WPE or PE values of each brain region between the aMCI and controls.

In order to determine whether the complexity of rsEEG can be a biomarker for detecting aMCI in diabetes, the correlations between neuropsychological test scores which significantly differ between control and aMCI and WPE (or PE) values from brain regions which significantly differ between control and aMCI were analysed. Pearson's linear correlation was employed for this analysis.

Bonferroni correction was used among the five regions for the $p$-value.

\section{Results}

The results showed that the values of WPE for rsEEG were lower in the aMCI diabetes group than those in the control group (see Figure 2).
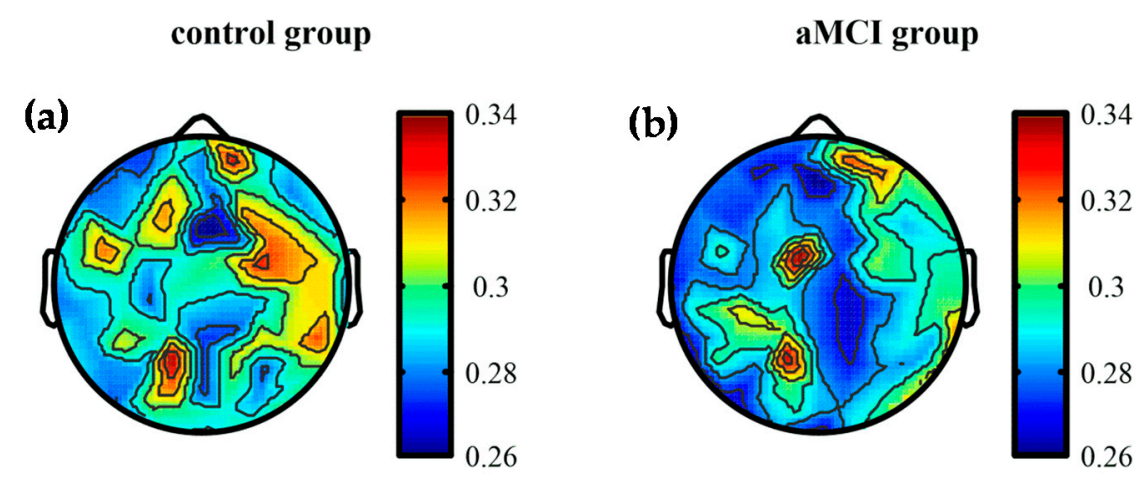

Figure 2. Normalized values of WPE in diabetics with controls (a) and aMCI (b) over the whole brain from group averages.

Figure 3 shows the comparative results for WPE values for the two groups in each brain region. The values of WPE in the RT region were significantly lower in the aMCI group than those in the control group (aMCI: $0.61 \pm 0.02$, control: $0.66 \pm 0.01, p=0.024$ ) before multiple testing among the five regions. No significant difference was found for the values of PE in all regions and WPE in other regions between the two groups whether they were corrected before or after. 


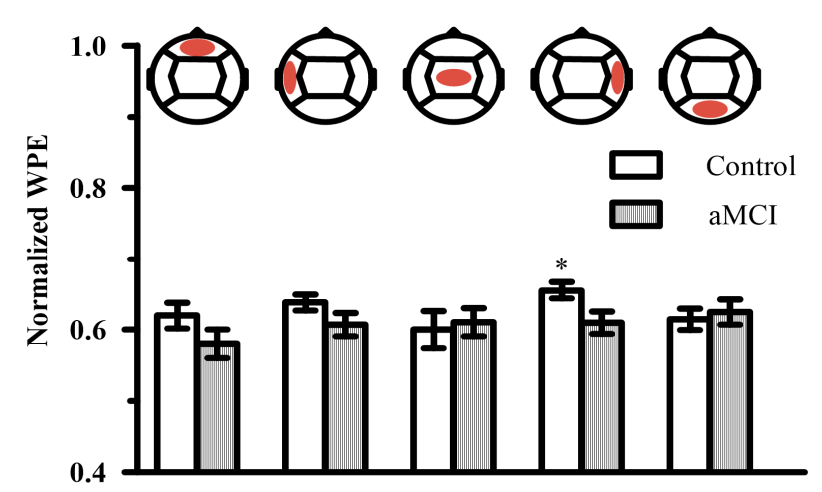

Figure 3. Comparison results for normalized WPE values for the two groups in each brain region. The values of WPE in the RT region were significantly lower in the aMCI group than those in the control group. $\left({ }^{*} p<0.05\right)$.

In order to further support the utility of WPE in detecting aMCI in diabetes, the correlations between the WPE values and neuropsychological assessment scores were analysed. The scores of MoCA, AVLT-Immediate recall, AVLT-Delayed recall, AVLT-Delayed recognition, BNT, and WAIS-DST were significantly different between the two groups, which was reported in [23]. The correlations between the normalized values of WPE in RT with these neuropsychological test scores are shown in Figure 4. The normalized values of the WPE in RT were positively correlated to the scores of AVLT-Immediate recall $(r=0.573, p=0.003)$, AVLT-Delayed recall $(r=0.569, p=0.004)$, AVLT-Delayed recognition ( $r=0.532, p=0.007)$, and WAIS-DST $(r=0.554, p=0.006)$. The $p$-value still smaller than 0.05 , thus the correlations were really significant.

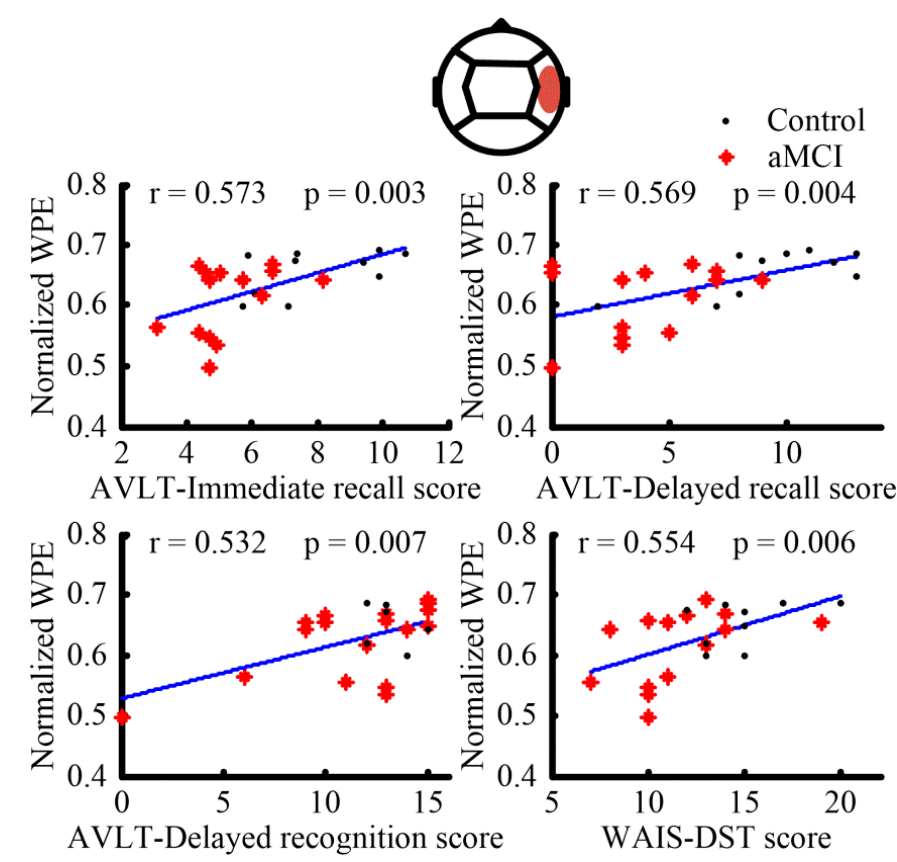

Figure 4. Analysis of correlations between the normalized WPE values in RT and the scores of AVLT-Immediate recall, AVLT-Delayed recall, AVLT-Delayed recognition, and WAIS-DST. They were all positively correlated.

\section{Discussion}

Since insulin-related effects may affect cognitive function [42], diabetes may accelerate the onset of MCI [24]. Other factors are impaired central glucose homeostasis and neurodegeneration due to 
the dysregulation of brain insulin signalling. Cerebrovascular damage (CVD) of the brain caused by diabetes [43] may result in increased risk of aMCI [44,45]. And type 2 diabetes may increase the risk of $\mathrm{aMCI}$ to $\mathrm{AD}$. Therefore, discovering and preventing aMCI in diabetics may reduce the risk of later AD onset.

In this study, we analysed the rsEEG of diabetics with aMCI and diabetic controls and compared signals from five brain regions by means of PE and WPE, which assess signal complexity. Several reports have demonstrated that biomarkers derived from EEG rhythms such as power spectral density, entropic complexity (especially PE and WPE), and other EEG features, were different among $\mathrm{MCI}, \mathrm{AD}$, and controls $[21,38,46]$, at least at the group level. Fernandez et al. showed that the Lempel-Ziv complexity of EEG signals is significantly reduced in MCI patients [47]. The features of regional complexity of EEG signals calculated using a support vector machine model could be non-invasive biomarkers to distinguish $\mathrm{MCI}$ and early $\mathrm{AD}$ [16]. Studies have shown that the entropy values of EEG were lower in MCI patients [48]. It has been demonstrated that the approximate entropy (ApEn) and sample entropy (SampEn) of EEG reduced significantly in MCI patients [49-53]; the value of multiscale entropy (MSE) decreased at short time scales and increased at long time scales in the frontal, temporal, and parieto-occipital regions $[49,54]$. It has been found that the PE values of rsEEG from MCI patients were significantly lower than those of controls in frontal, temporal, and anterior parietal regions [55]. However, PE does not take the effect of noise into account. WPE incorporates amplitude information and solves this problem [22]. WPE has been successfully used in distinguishing AD patients from normal healthy elders [38]. Their results showed that the WPE values of the AD group were lower than that of the control group at frontal and occipital regions in the delta band, for most regions in the theta band, and at frontal and central regions in the beta band. However, there has been no published work studying the usefulness of WPE in the MCI population.

This study explored the use of PE and WPE in detecting amnestic mild cognitive impairment in diabetes patients. We show that the values of WPE in the RT region are significantly lower in the aMCI group before Bonferoni correction, but there was no significant difference when using PE even before the correction. WPE has been demonstrated to be better than PE when measuring the complexity of signals in AD and the advantage of WPE over PE for investigating complexity abnormality in $\mathrm{AD}$ is proven [38]. WPE is an improvement of PE that incorporates the amplitude information of signals, which may be the reason for the different results between PE and WPE. Since there has been no previous studies using WPE on "general" MCI, our result regarding diabetic MCI can be considered a preliminary result, though the results are a little weak after Bonferroni correction. Further work will need to be performed to investigate the effectiveness of WPE on "general" MCI. The differences, in terms of brain regions, of our results from previous work may be due to the fact that patients were all diabetics (with or without aMCI), not healthy elders and AD patients without diabetes. With the richest connections to the hippocampus and a complex structure [56,57], signals collected from the temporal region are more sensitive to cognitive function, especially memory function, than other regions. Moreover, diabetes patients are commonly affected by CVD. These factors may have caused the differences in region-related results compared to previous studies.

Correlation analysis showed that WPE values in the RT region were positively and significantly correlated to the AVLT (immediate recall, delayed recall, delayed recognition) and the WAIS-DST. Since the sample was small, Figure 4 only shows the trends of the correlations of these patients, though most aMCI patients had the same or even higher WPE values than the control group. A larger sample is needed to confirm this result. Other significantly different neuropsychological assessment items were not correlated to the PE or WPE. Figure 4 suggests that the AVLT-delayed recall score seems to be extremely effective in separating the two groups, but for follow-up studies objective biomarkers are necessary. These correlations are just to confirm the feasibility and value of WPE as an rsEEG index for identifying aMCI among diabetics. The significant correlations of the AVLT and WAIS-DST with the WPE of rsEEG signals indicates that the WPE can distinguish aMCI from normal cognitive function in diabetics, at least at the group level. These results suggest that the rsEEG WPE in the RT region has 
clinical value in the early diagnosis and follow-up study of cognitive function impairment in diabetics, though it is only an EEG index, not a biomarker at present.

Using rsEEG WPE, early measures could be taken to mitigate the conversion process from aMCI to AD. Although this method cannot yet be used as a diagnostic tool in a clinical setting, the results at least show that this approach could detect aMCI effectively in the current sample of diabetic patients. The primary limitation of this study is the small sample size, so larger studies are needed to validate this method and support the conclusion.

Acknowledgments: This research was funded in part by the Natural Science Foundation of China (grant Nos. 61273063 and 81230023), Beijing Municipal Commission of Education, Hebei Municipal Commission of Education (GCC2014019), and China Postdoctoral Science Foundation (2015M572780).

Author Contributions: Zhijie Bian and Qiuli Li performed the experiments and acquired the data; Zhijie Bian analysed and interpreted the data for the study and drafted the manuscript; Gaoxiang Ouyang provided the methods for PE and WPE and revised the content and grammar; Zheng Li edited the manuscript; Lei Wang and Xiaoli Li designed the study, approved the final version to be published, and agreed to be accountable for all aspects of the study in ensuring that questions related to the accuracy or integrity of any part of the study are appropriately investigated and resolved; Xiaoli Li revised the manuscript critically for important intellectual content. All authors have read and approved the final manuscript.

Conflicts of Interest: The authors declare no conflict of interest.

\section{Abbreviations}

The following abbreviations are used in this manuscript:

$\begin{array}{ll}\text { EEG } & \text { Electroencephalo-graph } \\ \text { EGI } & \text { Electrical Geodesics Inc. } \\ \text { rsEEG } & \text { resting-state EEG } \\ \text { MMSE } & \text { minimum mental state examination } \\ \text { MoCA } & \text { montreal cognitive assessment } \\ \text { BNT } & \text { Boston Naming Test } \\ \text { AVLT } & \text { Auditory Verbal Learning Test } \\ \text { WAIS-DST } & \text { Wechsler Adult Intelligence Scale Digit Span Test } \\ \text { AD } & \text { Alzheimer's disease } \\ \text { MCI } & \text { mild cognitive impairment } \\ \text { aMCI } & \text { amnestic mild cognitive impairment } \\ \text { WPE } & \text { Weighted-permutation entropy } \\ \text { PE } & \text { Permutation entropy } \\ \text { ApEn } & \text { approximate entropy } \\ \text { SampEn } & \text { sample entropy } \\ \text { MSE } & \text { multiscale entropy } \\ \text { GESD } & \text { generalized extreme studentized deviate } \\ \text { CSF } & \text { cerebrospinal fluid } \\ \text { FDG-PET } & \text { fluorodeoxyglucose positron emission tomography } \\ \text { CVD } & \text { cerebrovascular damage } \\ \text { F } & \text { frontal } \\ \text { C } & \text { central } \\ \text { P } & \text { posterior } \\ \text { RT } & \text { right temporal } \\ \text { LT } & \text { left temporal } \\ & \end{array}$

\section{References}

1. Miles, W.R.; Root, H.F. Psychologic tests applied to diabetic patients. Arch. Intern. Med. 1922, 30, 767-777. [CrossRef]

2. Sejling, A.S.; Kjaer, T.W.; Pedersen-Bjergaard, U.; Diemar, S.S.; Frandsen, C.S.; Hilsted, L.; Faber, J.; Holst, J.J.; Tarnow, L.; Nielsen, M.N.; et al. Hypoglycemia-associated changes in the electroencephalogram in patients with type 1 diabetes and normal hypoglycemia awareness or unawareness. Diabetes 2015, 64, 1760-1769. [CrossRef] [PubMed]

3. Wang, R.; Wang, J.; Li, S.; Yu, H.; Deng, B.; Wei, X. Multiple feature extraction and classification of electroencephalograph signal for Alzheimers' with spectrum and bispectrum. Chaos 2015, 25, 013110. [CrossRef] [PubMed] 
4. Escudero, J.; Abasolo, D.; Hornero, R.; Espino, P.; Lopez, M. Analysis of electroencephalograms in Alzheimer's disease patients with multiscale entropy. Physiol. Meas. 2006, 27, 1091-1106. [CrossRef] [PubMed]

5. Gispen, W.H.; Biessels, G.J. Cognition and synaptic plasticity in diabetes mellitus. Trends Neurosci. 2000, 23, 542-549. [CrossRef]

6. Peila, R.; Rodriguez, B.L.; Launer, L.J. Type 2 diabetes, APOE gene, and the risk for dementia and related pathologies: The Honolulu-Asia aging study. Diabetes 2002, 51, 1256-1262. [CrossRef]

7. Morris, J.C. Mild Cognitive Impairment and Preclinical Alzheimer's Disease. Available online: http:/ / www.ncbi.nlm.nih.gov/pubmed/16025770 (accessed on 10 August 2016).

8. Seo, S.W.; Lee, J.H.; Jang, S.M.; Kim, S.T.; Chin, J.; Kim, G.H.; Kim, J.H.; Roh, J.H.; Kim, M.J.; Kim, S.H.; et al. Neurochemical alterations of the entorhinal cortex in amnestic mild cognitive impairment (aMCI): A three-year follow-up study. Arch. Gerontol. Geriatr. 2012, 54, 192-196. [CrossRef] [PubMed]

9. Strachan, M.W.; Reynolds, R.M.; Marioni, R.E.; Price, J.F. Cognitive function, dementia and type 2 diabetes mellitus in the elderly. Nat. Rev. Endocrinol. 2011, 7, 108-114. [CrossRef] [PubMed]

10. Petersen, R.C. Mild cognitive impairment as a diagnostic entity. J. Intern. Med. 2004, 256, 183-194. [CrossRef] [PubMed]

11. Schmitt, F.A.; Nelson, P.T.; Abner, E.; Scheff, S.; Jicha, G.A.; Smith, C.; Cooper, G.; Mendiondo, M.; Danner, D.D.; van Eldik, L.J.; et al. University of Kentucky Sanders-Brown healthy brain aging volunteers: Donor characteristics, procedures and neuropathology. Curr. Alzheimer Res. 2012, 9, 724-733. [CrossRef] [PubMed]

12. Albert, M.S.; DeKosky, S.T.; Dickson, D.; Dubois, B.; Feldman, H.H.; Fox, N.C.; Gamst, A.; Holtzman, D.M.; Jagust, W.J.; Petersen, R.C.; et al. The diagnosis of mild cognitive impairment due to Alzheimer's disease: Recommendations from the National Institute on Aging-Alzheimer's Association workgroups on diagnostic guidelines for Alzheimer's disease. Alzheimer's Dement. 2011, 7, 270-279. [CrossRef] [PubMed]

13. Takahashi, R.; Ishii, K.; Senda, M.; Ito, K.; Ishii, K.; Kato, T.; Makishi, Y.; Nishio, T.; Ikari, Y.; Iwatsubo, T. Equal sensitivity of early and late scans after injection of FDG for the detection of Alzheimer pattern: An analysis of 3D PET data from J-ADNI, a multi-center study. Ann. Nucl. Med. 2013, 27, 452-459. [CrossRef] [PubMed]

14. Toussaint, P.J.; Perlbarg, V.; Bellec, P.; Desarnaud, S.; Lacomblez, L.; Doyon, J.; Habert, M.O.; Benali, H. Resting state FDG-PET functional connectivity as an early biomarker of Alzheimer's disease using conjoint univariate and independent component analyses. NeuroImage 2012, 63, 936-946. [CrossRef] [PubMed]

15. Babiloni, C.; del Percio, C.; Lizio, R.; Marzano, N.; Infarinato, F.; Soricelli, A.; Salvatore, E.; Ferri, R.; Bonforte, C.; Tedeschi, G.; et al. Cortical sources of resting state electroencephalographic alpha rhythms deteriorate across time in subjects with amnesic mild cognitive impairment. Neurobiol. Aging 2014, 35, 130-142. [CrossRef] [PubMed]

16. McBride, J.C.; Zhao, X.; Munro, N.B.; Smith, C.D.; Jicha, G.A.; Hively, L.; Broster, L.S.; Schmitt, F.A.; Kryscio, R.J.; Jiang, Y. Spectral and complexity analysis of scalp EEG characteristics for mild cognitive impairment and early Alzheimer's disease. Comput. Methods Progr. Biomed. 2014, 114, 153-163. [CrossRef] [PubMed]

17. Cao, Y.; Tung, W.W.; Gao, J.B.; Protopopescu, V.A.; Hively, L.M. Detecting dynamical changes in time series using the permutation entropy. Phys. Rev. E 2004, 70, 046217. [CrossRef] [PubMed]

18. Dauwels, J.; Srinivasan, K.; Ramasubba Reddy, M.; Musha, T.; Vialatte, F.B.; Latchoumane, C.; Jeong, J.; Cichocki, A. Slowing and Loss of Complexity in Alzheimer's EEG: Two Sides of the Same Coin? Int. J. Alzheimer's Dis. 2011, 2011, 539621. [CrossRef] [PubMed]

19. Bandt, C.; Pompe, B. Permutation entropy: A natural complexity measure for time series. Phys. Rev. Lett. 2002, 88, 174102. [CrossRef] [PubMed]

20. Marsaglia, G.; Tsang, W.W.; Wang, J. Evaluating Kolmogorov's distribution. J. Stat. Softw. 2003, 8. [CrossRef]

21. Morabito, F.C.; Campolo, M.; Labate, D.; Morabito, G.; Bonanno, L.; Bramanti, A.; de Salvo, S.; Marra, A.; Bramanti, P. A longitudinal EEG study of Alzheimer's disease progression based on a complex network approach. Int. J. Neural Syst. 2015, 25, 1550005. [CrossRef] [PubMed]

22. Fadlallah, B.; Chen, B.; Keil, A.; Principe, J. Weighted-permutation entropy: A complexity measure for time series incorporating amplitude information. Phys. Rev. E 2013, 87, 022911. [CrossRef] [PubMed] 
23. Bian, Z.; Li, Q.; Wang, L.; Lu, C.; Yin, S.; Li, X. Relative power and coherence of EEG series are related to amnestic mild cognitive impairment in diabetes. Front. Aging Neurosci. 2014, 6. [CrossRef] [PubMed]

24. Roberts, R.O.; Knopman, D.S.; Geda, Y.E.; Cha, R.H.; Pankratz, V.S.; Baertlein, L.; Boeve, B.F.; Tangalos, E.G.; Ivnik, R.J.; Mielke, M.M.; et al. Association of diabetes with amnestic and nonamnestic mild cognitive impairment. Alzheimer's Dement. 2014, 10, 18-26. [CrossRef] [PubMed]

25. American Diabetes Association. Diagnosis and classification of diabetes mellitus. Diabetes Care 2012, 35, S64-S71.

26. World Medical Association. World medical association declaration of helsinki: Ethical principles for medical research involving human subjects. JAMA 2013, 310, 2191-2194.

27. Folstein, M.F.; Folstein, S.E.; McHugh, P.R. "Mini-mental state": A practical method for grading the cognitive state of patients for the clinician. J. Psychiatr. Res. 1975, 12, 189-198. [CrossRef]

28. Carlesimo, G.A.; Caltagirone, C.; Gainotti, G. The Mental Deterioration Battery: Normative data, diagnostic reliability and qualitative analyses of cognitive impairment. Eur. Neurol. 1996, 36, 378-384. [CrossRef] [PubMed]

29. Orsini, A.; Grossi, D.; Capitani, E.; Laiacona, M.; Papagno, C.; Vallar, G. Verbal and spatial immediate memory span: Normative data from 1355 adults and 1112 children. Ital. J. Neurol. Sci. 1987, 8, 537-548. [CrossRef]

30. Reitan, R.M. The Validity of the Trail Making Test as an indicator of organic brain damage. Percept. Motor Skills 1958, 8, 271-276. [CrossRef]

31. Novelli, G.; Papagno, C.; Capitani, E.; Laiacona, M.; Vallar, G.; Cappa, S.F. Three clinical tests for the assessment of lexical retrieval and production norms from 320 normal subjects. Arch. Psicol. Neurol. Psichiatr. 1986, 47, 477-506.

32. Lawton, M.P.; Brody, E.M. Assessment of older people: Self-maintaining and instrumental activities of daily living. Gerontologist 1969, 9, 179-186. [CrossRef] [PubMed]

33. Alagiakrishnan, K.; Zhao, N.; Mereu, L.; Senior, P.; Senthilselvan, A. Montreal Cognitive Assessment is superior to Standardized Mini-Mental Status Exam in detecting mild cognitive impairment in the middle-aged and elderly patients with type 2 diabetes mellitus. BioMed Res. Int. 2013, 2013, 186106. [CrossRef] [PubMed]

34. Nasreddine, Z.S.; Phillips, N.A.; Bedirian, V.; Charbonneau, S.; Whitehead, V.; Collin, I.; Cummings, J.L.; Chertkow, H. The Montreal Cognitive Assessment, MoCA: A brief screening tool for mild cognitive impairment. J. Am. Geriatr. Soc. 2005, 53, 695-699. [CrossRef] [PubMed]

35. American Psychiatric Association. Diagnostic and Statistical Manual of Mental Disorders (DSM); American Psychiatric Association: Washington, DC, USA, 1994.

36. Bandt, C. Ordinal time series analysis. Ecol. Model. 2005, 182, 229-238. [CrossRef]

37. Li, D.; Liang, Z.; Wang, Y.; Hagihira, S.; Sleigh, J.W.; Li, X. Parameter selection in permutation entropy for an electroencephalographic measure of isoflurane anesthetic drug effect. J. Clin. Monit. Comput. 2013, 27, 113-123. [CrossRef] [PubMed]

38. Deng, B.; Liang, L.; Li, S.; Wang, R.; Yu, H.; Wang, J.; Wei, X. Complexity extraction of electroencephalograms in Alzheimer's disease with weighted-permutation entropy. Chaos 2015, 25, 043105. [CrossRef] [PubMed]

39. Zhang, K.M.; Zhao, Z.Q. Selective Blockage by Yohimbine of Locus Coeruleus-Induced Inhibition of Nociceptive Reflex but Not That of C-Responses of Spinal Dorsal Horn Neurons in Rats. Acta Pharm. Sin. 1994, 15, 491-494.

40. Li, X.; Ouyang, G.; Richards, D.A. Predictability analysis of absence seizures with permutation entropy. Epilepsy Res. 2007, 77, 70-74. [CrossRef] [PubMed]

41. Seem, J.E. Using intelligent data analysis to detect abnormal energy consumption in buildings. Energy Build. 2007, 39, 52-58. [CrossRef]

42. Craft, S. Insulin resistance and Alzheimer's disease pathogenesis: Potential mechanisms and implications for treatment. Curr. Alzheimer Res. 2007, 4, 147-152. [CrossRef] [PubMed]

43. Debette, S.; Seshadri, S.; Beiser, A.; Au, R.; Himali, J.J.; Palumbo, C.; Wolf, P.A.; DeCarli, C. Midlife vascular risk factor exposure accelerates structural brain aging and cognitive decline. Neurology 2011, 77, 461-468. [CrossRef] [PubMed]

44. Knopman, D.S.; Roberts, R. Vascular risk factors: Imaging and neuropathologic correlates. J. Alzheimer's Dis. 2010, 20, 699-709. 
45. Roberts, R.O.; Kantarci, K.; Geda, Y.E.; Knopman, D.S.; Przybelski, S.A.; Weigand, S.D.; Petersen, R.C.; Jack, C.R., Jr. Untreated type 2 diabetes and its complications are associated with subcortical infarctions. Diabetes Care 2011, 34, 184-186. [CrossRef] [PubMed]

46. Sankari, Z.; Adeli, H.; Adeli, A. Wavelet Coherence Model for Diagnosis of Alzheimer Disease. Clin. EEG Neurosci. 2012, 43, 268-278. [CrossRef] [PubMed]

47. Fernandez, A.; Hornero, R.; Gomez, C.; Turrero, A.; Gil-Gregorio, P.; Matias-Santos, J.; Ortiz, T. Complexity analysis of spontaneous brain activity in Alzheimer disease and mild cognitive impairment: An MEG study. Alzheimer Dis. Assoc. Disord. 2010, 24, 182-189. [CrossRef] [PubMed]

48. Yang, A.C.; Wang, S.J.; Lai, K.L.; Tsai, C.F.; Yang, C.H.; Hwang, J.P.; Lo, M.T.; Huang, N.E.; Peng, C.K.; Fuh, J.L. Cognitive and neuropsychiatric correlates of EEG dynamic complexity in patients with Alzheimer's disease. Prog. Neuro-Psychopharmacol. Biol. Psychiatry 2013, 47, 52-61. [CrossRef] [PubMed]

49. Hornero, R.; Abasolo, D.; Escudero, J.; Gomez, C. Nonlinear analysis of electroencephalogram and magnetoencephalogram recordings in patients with Alzheimer's disease. Philos. Trans. R. Soc. Lond. A 2009, 367, 317-336. [CrossRef] [PubMed]

50. Abásolo, D.; Escudero, J.; Hornero, R.; Gómez, C.; Espino, P. Approximate entropy and auto mutual information analysis of the electroencephalogram in Alzheimer's disease patients. Med. Biol. Eng. Comput. 2008, 46, 1019-1028. [CrossRef] [PubMed]

51. Abasolo, D.; Hornero, R.; Espino, P.; Alvarez, D.; Poza, J. Entropy analysis of the EEG background activity in Alzheimer's disease patients. Physiol. Meas. 2006, 27, 241-253. [CrossRef] [PubMed]

52. Abasolo, D.; Hornero, R.; Espino, P.; Poza, J.; Sanchez, C.I.; de la Rosa, R. Analysis of regularity in the EEG background activity of Alzheimer's disease patients with Approximate Entropy. Clin. Neurophysiol. 2005, 116, 1826-1834. [CrossRef] [PubMed]

53. Woon, W.L.; Cichocki, A.; Vialatte, F.; Musha, T. Techniques for early detection of Alzheimer's disease using spontaneous EEG recordings. Physiol. Meas. 2007, 28, 335-347. [CrossRef] [PubMed]

54. Kim, K.; Kim, C.-H.; Cichocki, A.; Kim, S.; Park, J.-H. Multiscale Entropy Analysis of Eeg from Patients under Different Pathological Conditions. Fractals 2007, 15, 399-404.

55. Timothy, L.T.; Krishna, B.M.; Menon, M.K.; Nair, U. Fractals, Wavelet, and Their Applications; Bandt, C., Barnsley, M., Devaney, R., Falconer, K.J., Kannan, V., Vinod Kumar, P.B., Eds.; Springer: Berlin/Heidelberg, Germany, 2014; pp. 395-406.

56. Johnson, J.D. The conversational brain: Fronto-hippocampal interaction and disconnection. Med. Hypotheses 2006, 67, 759-764. [CrossRef] [PubMed]

57. Moretti, D.V.; Miniussi, C.; Frisoni, G.B.; Geroldi, C.; Zanetti, O.; Binetti, G.; Rossini, P.M. Hippocampal atrophy and EEG markers in subjects with mild cognitive impairment. Clin. Neurophysiol. 2007, 118, 2716-2729. [CrossRef] [PubMed]

(C) 2016 by the authors; licensee MDPI, Basel, Switzerland. This article is an open access article distributed under the terms and conditions of the Creative Commons Attribution (CC-BY) license (http://creativecommons.org/licenses/by/4.0/). 